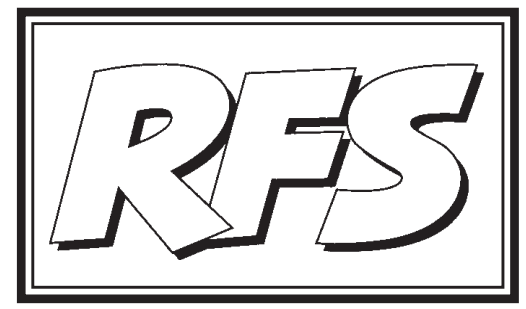

Revista de Fomento Social, 61 (2006), 423-453

\title{
La contabilidad de gestión en las cooperativas de crédito. Una aproximación a la imputación de costes
}

(Palabras clave: Cooperativas de cRÉdito, Costes, Actividades, Contabilidad de GESTIÓN.

KEY WORDS: CREDIT CO-OPERATIVES, COSTS, ACtIVITY, MANAGEMENT ACCOUNTING)

1 Universidad Miguel Hernández. Escuela Politécnica Superior de Orihuela. Departamento Economía Agroambiental. Carretera de Beniel, km. 3,2; 03312 Orihuela (Alicante); Tfo: + 34 966749636; E-mail: amparo.melian@umh.es; Catedrática de Escuela Universitaria. Profesora de Economía Agraria.

La autora agradece a los dos revisores anónimos las correcciones y recomendaciones efectuadas, las cuales han permitido mejorar la versión inicial del trabajo. 


\section{Las cooperativas de crédito como integrantes del sistema financiero español}

En el actual contexto financiero de elevada competencia, las cooperativas de crédito (cajas rurales y cajas populares y profesionales) rivalizan con bancos y cajas de ahorro en el negocio de la intermediación y prestación de servicios financieros. Aunque son las menos significativas en cuanto a peso relativo por instituciones, $4 \%$ sobre balance consolidado del conjunto de entidades de crédito (Banco de España, 2006), son las que más han visto incrementada su actividad (16\% frente al 14\% de cajas de ahorro y $10 \%$ de bancos).

Las cooperativas de crédito son empresas financieras que actúan en un entorno globalizado, competitivo y cambiante, y que junto con los bancos y cajas de ahorro constituyen el denominado sistema bancario, y cuya finalidad principal es la canalización de los flujos financieros entre prestamistas y prestatarios (Rodriguez, et al., 2003). Son entidades bancarias de ámbito territorial por motivo de su origen geográfico, con independencia de su mayor o menor expansión hacia otras demarcaciones. Hasta hace unos años y debido a que el mercado estaba claramente segmentado han gozado de cierto monopolio en sus mercados locales (Fuentelsaz, et al., 2003). Su modelo de negocio se centra en la banca al por menor, intensiva en oficinas y con patrones de actividad similares a los de las cajas de ahorro. La principal diferencia de las cooperativas de crédito con respecto al resto de intermediarios bancarios reside en la forma jurídica, sociedad cooperativa frente a sociedad anónima (caso de los bancos) o fundaciones (caso de las cajas de ahorros), y en su finalidad.

Sus objetivos van más allá de la simple obtención de beneficios y su componente social es importante, pero también lo es que, dado que realizan su actividad en un contexto en el que compiten con otros intermediarios financieros que no están sujetos a esos principios, su supervivencia sólo estará garantizada en la medida en que puedan alcanzar unos niveles de eficiencia similares a los de sus rivales (Fuentelsaz, et al., 2003).

Las cooperativas de crédito, como el resto de sociedades cooperativas se gobiernan siguiendo la estructura tradicional cooperativa cumpliendo con el principio de puertas abiertas, libre adhesión de socios, y control democrático. Se las identifica como organizaciones empresariales adscritas al extenso y variado ámbito de la denominada economía social (Barea y Monzón, 2002). Tienen una clara orientación hacia sus socios y clientes, y 
gozan de una cultura empresarial propia basada en los principios cooperativos y de gestión democrática que las identifican y distinguen frente a otras formas jurídicas (García-Gutiérrez, 1995). La fórmula cooperativa se convierte en la respuesta idónea para resolver determinados problemas (Morales, 2005). El cumplimiento de los principios cooperativos, como valores de su idiosincrasia sobre todo en lo que hace referencia a la educación y a la participación democrática supone un desafío crucial para mantener la adhesión de sus miembros y su implicación en la gestión de la entidad (Pedelty, 1999).

Sus características específicas hacen de esta modalidad cooperativa y de entidad de crédito todo un referente, sobre todo en las zonas donde principalmente han desarrollado su actividad, pues las cooperativas de crédito han manifestado una especialización funcional y territorial, aunque actualmente también expanden su actividad hacia otros ámbitos alejados del negocio tradicional. De igual forma hacia los colectivos menos atractivos para el negocio bancario. Así, Palomo y Valor (2001) constatan una especialización en la financiación de los estratos de renta baja (43\%) y media-baja (37\% del total de sus clientes), mientras que los grupos de renta media-alta y alta suponen un porcentaje de clientes inferior (del $12 \%$ y $5 \%$ respectivamente). Esto de algún modo viene a ratificar la capacidad de inclusión financiera de estas entidades, definida como la aptitud de una entidad para conectar con los circuitos financieros a determinados agentes económicos, que por diversas razones (económicas, distancia, sexo, etc.) se hallan excluidos de éstos. Según Chaves y Soler (2004) este concepto es inherente a las cooperativas de crédito, pues su labor ha permitido históricamente conectar la capacidad o la necesidad de financiación de determinados sectores con los circuitos financieros de la economía, lo que ha contribuido a su dinamización y su vinculación con el resto de sectores.

De las 269 entidades de crédito operativas en España en 2005, bancos, cajas de ahorro y cooperativas de crédito, 83 pertenecen a este último tipo. La especialización que caracteriza el cooperativismo de crédito en España se manifiesta en el tipo de entidades que lo componen: cajas rurales (las más numerosas, con 77 entidades), cajas populares y cajas profesionales (6 entidades), según la orientación principal de su actividad hacia un determinado sector u otro. Con el sector agrario se han vinculado estrechamente las cajas rurales, pues tradicionalmente se han ocupado de la financiación del medio rural, aunque cada vez más están ampliando su actividad hacia otros sectores. El peso de estas entidades sobre el total del sistema finan- 
ciero medido por volumen de activos es bajo (4\%) (Cuadro 1), sin embargo el crecimiento del balance es el más elevado entre las tres entidades. El beneficio neto supera los 600 millones de euros lo que representa el 4,5\% del beneficio total del conjunto de entidades de crédito, el ROA (beneficio neto sobre activos) ha sido del $0,8 \%$, la misma cifra que en la banca y algo superior a la de las cajas de ahorro.

\section{CUADRO 1. Principales datos económicos del sistema financiero español} por instituciones

\begin{tabular}{|l|c|c|c|r|c|c|}
\hline Instituciones & $\begin{array}{c}\text { Volumen } \\
\text { activo }\end{array}$ & $\begin{array}{c}\text { Peso relativo } \\
\text { s/ Vol. Activo }\end{array}$ & $\begin{array}{c}\text { \% Crecimiento } \\
\text { balance }\end{array}$ & $\begin{array}{c}\text { Beneficio } \\
\text { neto }\end{array}$ & $\begin{array}{c}\text { Peso relativo } \\
\text { s/ B } \text { B Neto }^{\circ}\end{array}$ & ROA \\
\hline Bancos & 1.169 .147 & 56,5 & 10,3 & 7.827 & 58,0 & 0,8 \\
\hline Cajas Ahorro & 816.820 & 39,5 & 14,3 & 5.063 & 37,5 & 0,7 \\
\hline Coop. Crédito & 82.180 & 4,0 & 16,0 & 610 & 4,5 & 0,8 \\
\hline
\end{tabular}

Fuente: elaboración propia a partir de Banco España, 2006.

${ }^{1}$ Datos en millones de euros a 31 diciembre 2005.

Las 83 cooperativas de crédito disponen a 31 de diciembre de 2005 de 4.656 oficinas y el número de empleados asciende a 17.650 , lo que supone respectivamente una cuota del 11 y $7 \%$ sobre el total de entidades de crédito, el número de cajeros automáticos es de aproximadamente 4.200 (3\% de variación con respecto al año anterior), y el de socios de más de 1.670 .000 (más de un 6\% de incremento) (Banco de España, 2006).

\section{El output en la empresa bancaria. El problema de su delimitación}

Íntimamente relacionado con la función de producción en el sistema bancario, lo cual valida la importancia del control de gestión en la misma, es la coincidencia en el espacio y tiempo de los procesos de producción y consumo (Perdiguer, 1991). Además, se producen con periodicidad y carácter cíclicos momentos puntuales con exceso de demanda, que no pueden ser atendidos con una producción previa de stocks al ser servicios financieros lo que se demanda y no productos de consumo. 
Como empresas de servicios ${ }^{2}$, su peculiaridad consiste en vender productos intangibles ${ }^{3}$. La intangibilidad de éstos y su caducidad pueden plantear dificultades de acoplamiento entre la oferta y la demanda en determinadas épocas. Así pues, su output no se obtiene a través de un proceso productivo industrial (aprovisionamiento de materias primas, transformación-fabricación y distribución). Las características propias de los outputs en las empresas de servicios son (Ibarrondo, 2002a): a) la intangibilidad de ciertos componentes, b) la inseparabilidad entre la producción del servicio y su consumo, c) la heterogeneidad, lo que dificulta su estandarización, afectando susceptiblemente la personalidad del que lo presta o lo solicita, y d) la caducidad, pues el consumo realizado se efectúa simultáneamente a la producción, no siendo almacenables los productos. Todas estas características las presentan los productos bancarios, aunque particularmente la intangibilidad es función del producto en sí, ya que algunos de ellos como el alquiler de las cajas de seguridad o ciertos productos de activo y pasivo, concesión de un préstamo o captación de fondos, gozan de cierta tangibilidad que les permite ser comparados con los de las empresas competidoras, no así los de servicio puro como asesoría o gestión.

En la empresa bancaria la oferta de las instituciones se concreta en una oferta de servicios destinados a atender al cliente (Barroso, 1995). El contacto directo entre el personal de la empresa y el cliente es muy importante, así como el tiempo dedicado a fomentar estas relaciones. Las operaciones de transformación se apoyan en el personal y en las actividades de front office, constituyendo el propio proceso el centro de atención en la prestación del servicio.

Aunque la producción se suele identificar con la transformación técnica de un determinado conjunto de bienes, en sentido amplio comprende todas las actuaciones encaminadas a lograr un incremento de la utilidad de los objetivos económicos para satisfacer las necesidades humanas. Los intermediarios financieros bancarios efectúan una verdadera transformación de activos, dado que adquieren instrumentos financieros primarios con sus características de riesgos y plazos, suministrando fondos a los agentes económicos que los

2 Según O. B. MARTINSON (1994) las empresas de servicios desarrollan una actividad económica mediante la cual obtienen un output que en esencia no tiene entidad física pero que suministrado proporciona cierta utilidad para el cliente en forma de elementos intangibles.

3 Sobre el concepto de bien y servicio, y tipos de productos, I. Grande (1996) y P. KotLer (1992). 
solicitan, y emiten instrumentos secundarios a distintos plazos gracias a los cuales captan los recursos que necesitan para su actividad intermediadora, creando instrumentos que son aceptados entre los ahorradores. La gestión del tiempo es lo que permite adecuar las preferencias entre ahorradores e inversores.

La aplicación de los fondos captados al proceso productivo no modifica la naturaleza de los recursos sino su grado de liquidez. Además, a diferencia de otras empresas, los inputs (fondos captados) no son adquiridos en propiedad (como las materias primas en las empresas industriales) sino que se obtienen en depósito a cambio de una retribución. La entidad de depósitos es una empresa de servicios, y como tal no efectúa una transformación intrínseca de la naturaleza de ciertos bienes -materia prima-para la obtención de otros -productos- (Ibarrondo, 2002c).

Sánchez Asiain (1992) expresa que la materia prima básica de la empresa bancaria es la información, afirmando que la banca realiza una función de intermediación entre ahorradores y prestatarios en la que genera un valor añadido. En ese proceso de intermediación administra y transforma información, su materia prima básica, que le sirve para reducir (mediante la diversificación) el riesgo conjunto de sus inversiones, convertirse en tomadora de riesgo de última instancia, reconducir y equilibrar los desfases temporales de los flujos financieros de la economía, y asegurar la confianza en el sistema.

En esta línea es muy importante considerar las dificultades que entraña la identificación del output bancario, aspecto común a otras empresas de servicios, sobre todo por la naturaleza intangible propia de algunos productos, o por la dificultad de medir y cuantificar a efectos de coste, otros aspectos y variables como pueden ser la calidad del servicio prestado o el tiempo de respuesta a las necesidades del cliente. Disponer de un catálogo de productos que constituyan objetos de cálculo adecuados para la gestión y para la toma de decisiones desde los diversos eslabones jerárquicos no resulta sencillo.

Según Ibarrondo (2002a) la actividad desempeñada por las entidades de depósitos se traduce en la prestación de diversos servicios, básicamente financieros, por lo que su proceso productivo puede catalogarse de producción múltiple y en función de la tipología del proceso, de producción conjunta, pues se da una interdependencia entre los productos, fruto de la naturaleza del propio proceso productivo. Esta interdependencia puede ser debida a la 
propia demanda, pues el cliente elige y vincula determinados productos, o a la función de producción, por el uso combinado de ciertos productos.

La problemática de la delimitación del output bancario se pone de manifiesto no sólo por la inexistencia de un producto almacenable o el suministro de productos interdependientes, sino por la posibilidad de considerar los fondos captados como un input o como un output de la transformación bancaria ${ }^{4}$, o incluso confiriéndole un carácter dual ${ }^{5}$.

Sealey y Lindley (1977) consideran que la ausencia de una delimitación clara entre los aspectos técnicos y económicos del proceso productivo bancario es la causa de que no exista unanimidad en la determinación del output en la producción bancaria. Así, desde la perspectiva técnica el output bancario lo constituye el flujo de servicios suministrados a los clientes, tanto a los depositantes como a los prestatarios. Pero desde la perspectiva económica sólo son outputs los servicios susceptibles de generar un valor de mercado superior a los inputs utilizados para su obtención.

Si se adopta el criterio de producción bancaria según Sealey y Lindley (1977) atendiendo al criterio técnico, se establecen dependiendo de los tres subprocesos productivos, captación de fondos, colocación de fondos y prestación de servicios puros, las siguientes tres categorías de output bancario: productos de pasivo ${ }^{6}$, productos de activo y productos de servicio puro. El cuadro 2 presenta una clasificación de los productos bancarios atendiendo a estas tres categorías.

4 Sobre la concepción del output bancario y su problemática G.J. BENSTON (1972), W.F. MACKARA (1975), O. Fanjul y F. Maravall (1985), J. Revell (1988), F. Perez y J.M. Pastor (1994), F. Perez y J. Quesada (1991), J. Quesada (1994), J.A. Novo y R. VARela (1996).

5 D. PRior y V. Salas (1994).

6 Desde el punto de vista económico Sealey y Lindley consideran que su clasificación como producto resulta dudosa. 
CUADRO 2. Tipología de los productos bancarios

\begin{tabular}{|c|c|c|c|}
\hline Tipo & Fundamento & Productos & Característica \\
\hline Pasivo & $\begin{array}{l}\text { Captación } \\
\text { de ahorro }\end{array}$ & $\begin{array}{l}\text { Depósitos: cuentas corrien- } \\
\text { te, cuentas de ahorro, impo- } \\
\text { siciones a plazo. } \\
\text { Cesión temporal de activos. } \\
\text { Emisión de títulos: Valores } \\
\text { negociables. } \\
\text { Participaciones de activo. }\end{array}$ & $\begin{array}{l}\text { Implican un } \\
\text { coste }\end{array}$ \\
\hline Activo & $\begin{array}{l}\text { Inversión de } \\
\text { fondos }\end{array}$ & $\begin{array}{l}\text { Préstamos y cuentas de } \\
\text { crédito. } \\
\text { Cartera de valores: renta } \\
\text { fija, renta variable. } \\
\text { Arrendamiento financiero y } \\
\text { factoring. }\end{array}$ & $\begin{array}{l}\text { Generan un } \\
\text { rendimiento }\end{array}$ \\
\hline $\begin{array}{l}\text { Servicio } \\
\text { puro }\end{array}$ & $\begin{array}{l}\text { Prestación } \\
\text { de un } \\
\text { servicio }\end{array}$ & $\begin{array}{l}\text { Gestión de pagos y cobros: } \\
\text { domiciliaciones, transferen- } \\
\text { cias y tarjetas de crédito. } \\
\text { Asesoramiento financiero. } \\
\text { Gestión patrimonios. } \\
\text { Comercialización fondos: } \\
\text { inversión, pensiones. } \\
\text { Comercialización seguros. } \\
\text { Intermediación: compra- } \\
\text { venta valores mobiliarios. } \\
\text { Otras operaciones: mercado } \\
\text { de opciones y futuros. }\end{array}$ & $\begin{array}{l}\text { Generan un } \\
\text { rendimiento }\end{array}$ \\
\hline
\end{tabular}

Fuente: Elaboración propia.

Las tareas que se desarrollan en cada subproceso para la contratación o formalización de un servicio bancario pueden ordenarse secuencialmente en cinco fases (Ibarrondo, 2002a): 1) contacto o entrevista con el cliente, 2) recogida de información en relación a la operación, 3) estudio y análisis de la información, 4) toma de decisiones y sanción de la operación, 5) formalización y contratación, para a continuación dar lugar a la prestación en sentido propio del servicio y a su seguimiento posterior. 
No obstante y a pesar de que se han delimitado tres subprocesos ${ }^{7}$, éstos están íntimamente relacionados de modo que la separabilidad entre ellos, aunque se haya definido, no está exenta de cierta interdependencia. Así pues, actuaciones del subproceso de prestación de servicios están vinculadas al proceso de captación de fondos, en la medida en que un producto de pasivo, como puede ser una cuenta corriente, es un instrumento soporte de cobros y pagos, pudiendo ordenar transferencias a su cargo o efectuar domiciliaciones, emplear tarjetas de crédito o débito. También se da una estrecha vinculación entre el subproceso de captación de fondos y el de colocación, dado que los recursos financieros obtenidos en el subproceso de captación de fondos constituyen un input específico para el de colocación. Además muchos de los factores productivos de una entidad de depósitos: personal, instalaciones-infraestructuras, equipos informáticos, etc. son compartidos por las distintas operaciones que tienen lugar en dichos subprocesos.

Cuando se presta un servicio se genera un output de carácter intangible (el servicio prestado) sobre el que resulta más difícil identificar los costes ya que no existe un soporte (físico) un producto sobre el que recaigan los consumos de recursos. Quien demanda el consumo de recursos es la prestación de un servicio que consiste por si mismo en el desempeño de una o varias actividades (Ruiz y Tirado, 2004b).

Por otra parte en las empresas bancarias existe un elemento diferencial en su actividad que no puede soslayarse, la relación que se establece con la clientela. Es importante el manejo de información, la gestión del capital intelectual, la formación de consultores, unos adecuados métodos de trabajo, en definitiva aspectos intangibles que añaden valor al servicio, y que permiten detectar oportunidades de benchmarking interno. Los empleados, los socios y los clientes valoran estos activos intagibles, tan difíciles de cuantificar y comercializar, pero que son los que en definitiva avalan la reputación externa de la empresa.

\section{La contabilidad de gestión y los modelos tradicionales de imputación de costes}

Ante la actual situación del mercado financiero donde se desenvuelve la actividad de las cooperativas de crédito, estrechamiento del margen

7 Esta clasificación está habitualmente aceptada en el sector bancario. 
financiero, relativos pocos ingresos derivados de los otros productos, incremento de la competencia, liberalización bancaria, desintermediación, descentralización, aumento de la cultura financiera de los clientes, mayor poder de negociación de éstos, etc., se hace necesario un estricto control de los costes que afectan a los procesos productivos de estas entidades, a fin de asegurar su permanencia en el mercado.

La contabilidad de gestión es un instrumento al servicio de la toma de decisiones $^{8}$ para cada nivel jerárquico y debe cumplir con el requisito de economicidad. Su desarrollo se debe entre otros, a la limitada información que aporta la contabilidad financiera para efectuar análisis internos y externos de la gestión. Los instrumentos metodológicos de esta disciplina han ido perfeccionándose para adaptarse a las necesidades de información de los usuarios internos (Vera, 1997).

La competencia aumenta la necesidad de un coste preciso del producto porque una empresa no puede trasladar sus ineficacias en forma de precios más elevados (Brimson, 1995). El control de costes será una herramienta informativa básica que permita valorar la eficacia alcanzada por los distintos factores productivos. El conocimiento de los costes incurridos para su posterior racionalización es fundamental para incrementar o cuanto menos mantener ese nivel de competitividad.

Según Mateos (2002) el actual desarrollo de la contabilidad de costes se debe a la necesidad de instrumentar el proceso de toma de decisiones en base a una información próxima a la realidad, que minimice el riesgo e identifique y recoja de forma rápida y sistemática los cambios que se produzcan, permitiendo adaptar la organización a las continuas modificaciones de su entorno y reduciendo la posibilidad de adopción de decisiones erróneas.

Previamente al desarrollo de la contabilidad de costes es interesante recordar la definición que de los mismos realiza Blanco (2002) donde los define como la medida y valoración del consumo realizado o previsto por la aplicación racional de los factores productivos para la obtención de un producto, trabajo o servicio, de modo que este consumo incremente el valor del producto o servicio.

8 Un estudio en profundidad sobre contabilidad de gestión y los sistemas ABC y ABM en P. TiRADO (2003), Gestión de costes y mejora continua. Los sistemas de costes y de gestión basados en las actividades ( $A B C-A B M)$, Colección ETEA, Ed. Desclée de Brouwer. 276 pp. 
La dificultad en la asignación de ciertos costes indirectos y el hecho de que los diversos procesos productivos en la empresa no son acometidos del mismo modo, y conllevan un consumo distinto de costes hace que los sistemas de costes tradicionales pierdan vigencia al no reconocer las diferencias específicas de las diversas actividades productivas. En esta línea Forrest (1996) afirma que: "los sistemas tradicionales de contabilidad de costes no proporcionan una adecuada información operacional a la dirección... ignorando la interrelación entre las actividades..., siendo la interdependencia de actividades la clave para mejorar la productividad y calidad en la organización, adoptando una mejora continua".

La idea básica del sistema de costes por actividades es que la actividad consume recursos y los productos consumen actividades. La actividad se define (AECA, 1998) como un conjunto de actuaciones o de tareas que tienen por objeto la obtención de un output (producto o servicio), mediante el consumo de una serie de factores o inputs, que son consecuencia tanto de la concepción de las tareas como de la frecuencia con que éstas deben ser acometidas.

La metodología más aceptada es la que considera que los sistemas $\mathrm{ABC}$ ("Activity Based Costing" o Costes Basados en las Actividades) se desarrollan en dos etapas: la primera, la determinación del coste de las actividades, y la segunda, la imputación de esos costes a los objetivos de costes en función de la relación existente entre cada actividad y el objetivo final de coste. En ambos casos, la asignación de los costes debe hacerse teniendo en cuenta la relación causa-efecto que existe entre los costes (efecto) y las actividades que causan dichos costes, así como entre los objetos de coste y las actividades demandadas por éstos (Tirado, 2003). El sistema de costes por actividades ha sido estudiado en diversos sectores económicos, entre ellos el financiero, Fuentelsaz y Gomez (2001), y García (2001).

Tras el desarrollo inicial de los sistemas de costes basados en las actividades, las empresas en la década de los 90 utilizan las actividades definidas por el sistema $\mathrm{ABC}$ como instrumento de análisis en la gestión empresarial. La empresa se percibe como un conjunto de actividades que consumen recursos y que han de ser gestionadas de forma que se favorezca la mejora continua y se satisfagan las expectativas de los diferentes grupos de interés, dando lugar a los sistemas de gestión basados en las actividades, ("Activity Based Management") o ABM. Este sistema se enmarca dentro de un proceso de mejora continua en el que se analiza los procesos y las variables claves 
para el éxito más allá de los costes (calidad, tiempo, valor añadido, innovación, etc.) (Ruiz y Tirado, 2004b).

El sistema de costes basado en las actividades parte de la desagregación de la empresa en las unidades o centros en los que se estructura la unidad de negocio (De la Cuesta, 1996). La actividad sería pues lo que la empresa hace en esa unidad o centro, y se identificará a partir de una primera agregación de tareas. Posteriormente la asignación de costes se podrá efectuar de forma directa cuando exista una vinculación clara o se deberá contemplar un "reparto" basado en una clave según el consumo que se haya efectuado de un determinado factor. La clave de reparto se constituye como un instrumento que permite la distribución de los elementos de coste entre las actividades a las que sea asignable y está basada en un factor cuya medida es fácilmente determinable y cuyo valor se supone proporcional, aproximadamente, al verdadero consumo de costes (AECA, 1993). La gestión por actividades no exige ningún cambio de organización. Sin embargo, la luz que aporta a las actividades y procesos puede incitar a modificar la organización para una mejor consideración de sus principales retos (Lorino, 1993).

Las dos principales ventajas de la contabilidad por actividades para una empresa son, según Brimson (1995), que permite obtener un coste del producto preciso, y que posibilita una mejor percepción de las oportunidades para la reducción de costes y la mejora del rendimiento. Dicho autor define el coste de una actividad como el gasto total de todos los factores de producción imputables que se asignan para realizar una actividad. Y son centros de actividad los segmentos del proceso de producción para los cuales se requiere un conocimiento independiente de las actividades realizadas. Son centros de responsabilidad donde la información de las funciones concretadas en actividades proporciona a los diferentes niveles jerárquicos de la organización información relevante para un mayor control.

A modo de síntesis recordamos que el cálculo de costes en los sistemas tradicionales estaba enfocado hacia el control, mientras que en la concepción actual de análisis de actividades está enfocado hacia la gestión estratégica de costes y a la mejora de la eficiencia de los procesos. En la nueva metodología se evita la vinculación directa entre el coste y el objetivo final de una operación, pues el causante del coste no es el elemento final de la operación sino la realización de determinadas actividades y el empleo de ciertos factores. Lo importante ahora es medir la eficiencia en el consumo de factores de producción. Primero se reorienta el cálculo de costes hacia las actividades y después se agregan éstas a nivel de procesos, productos o clientes. 
El sistema de actividades proporciona un mejor conocimiento de la empresa y sus procesos, lo que facilita en gran medida el proceso de toma de decisiones y en consecuencia la planificación, entendida como el proceso de conexión entre los objetivos, estrategias, políticas y decisiones en la empresa.

Algunos autores como Lorino (2003) definen la gestión por actividades más que como un instrumento formal como una nueva filosofía de gestión. Según Castelló y Lizcano (1994) toda la información necesaria para implantar el sistema de costes por actividades suele estar ya disponible por lo que el coste de medición constará de dos elementos básicos: el coste de la rutina informativa generada por el propio sistema de costes y el coste derivado de la aplicación de los cálculos necesarios para determinar el coste del objetivo final.

Con el modelo $\mathrm{ABC}$ se determina el coste completo de un producto agregando el coste de las actividades a distintos niveles con base en las relaciones de causalidad entre actividades y productos. Así, no sólo se determina el valor final del coste, sino que también se conocerá la aportación de cada clase de coste en función de la actividad, y en definitiva, la contribución de cada una de las actividades al valor añadido del producto.

En las entidades bancarias es importante disponer de un sistema de contabilidad de gestión que proporcione información sobre el coste de cada servicio y permita conocer la contribución al resultado de cada línea de servicios. La suma de negocios que hoy caracterizan el sector exige un análisis específico de cada uno de ellos, restando importancia a un análisis global basado en estados financieros globales. El control de gestión (AMAT, 1992) no es sólo un sistema para medir resultados sino un instrumento capaz de orientar e influir en que el comportamiento individual y organizativo sea el más conveniente para alcanzar los objetivos de la empresas.

\section{Una aproximación a la imputación de costes por actividades en las cooperativas de crédito}

La estrategia de banca universal se caracteriza por la obtención de forma conjunta de varios productos. La función de producción integrada dificulta la correcta asignación de costes, dado que según Bengoechea y Pizarro (1993) en el sector bancario aproximadamente el $80 \%$ de los costes de una entidad son costes compartidos por las distintas actividades desarrolladas. Esto ha 
podido propiciar que tradicionalmente no se realizara un efectivo control de los costes y que se desconociese la contribución individual de cada uno de los productos al resultado global, toda vez que se producían a su vez mecanismos de subsidiariedad.

El desarrollo de la contabilidad de gestión en las entidades de depósito se ha producido con un importante retraso respecto a las empresas industriales, no sólo en España sino también en los países de nuestro entorno (Ibarrondo, $2002 b)$. No obstante su importancia y necesidad de implantación en las entidades financieras no se discute pues la Circular $\mathrm{n}^{\mathrm{0}} 4 / 1991$, en su norma sexta apartado 4 indica que: "las entidades establecerán una contabilidad analítica que aporte información suficiente para el cálculo de los costes y rendimientos de los diferentes productos, servicios, centros, departamentos, líneas de negocio y otros aspectos que interesen a la gestión de su negocio".

AECA (1995) define la contabilidad de gestión bancaria como "la rama de la contabilidad que tiene por objeto la captación, medición y valoración de la circulación interna, así como su racionalización y control, con el fin de suministrar a la organización bancaria la información relevante para la toma de decisiones, la planificación y el control".

La contabilidad de gestión en las entidades financieras debe ser capaz de proporcionar información sobre los costes y rendimientos de las líneas de productos, las oficinas-sucursales o centros de actividad, y los distintos segmentos de clientes, facilitando la planificación y control de las actividades, y la toma de decisiones. También permite identificar las relaciones causa-efecto (eslabones) en el desarrollo de los procesos, abandonando la visión vertical de la organización.

Las decisiones en la entidad bancaria deben estar apoyadas en sistemas de información ágiles y flexibles, que permitan respuestas rápidas. Este sistema de información bien puede ser la contabilidad de gestión que como indica AECA (1995) debe suministrar información relevante sobre aspectos tales como: "abrir o cerrar una oficina o una unidad de negocio; producir internamente o subcontratar fuera; medir los márgenes de los procesos de los centros, de los mercados, de los clientes o de los productos; medir riesgos como el de interés o de mercado, de insolvencias o de liquidez; o proporcionar criterios para evaluar la eficacia de los servicios centrales o de cualquier actividad bancaria reflejada en el balance o fuera de él".

El control de los costes de transformación-explotación es muy importante en estas entidades dada la evolución experimentada por el mercado como 
consecuencia de la reducción de los márgenes de intermediación, y otros factores como el incremento de la competencia o el proceso de innovación financiera. Conocer los costes operativos por productos, oficinas y clientes va a ser fundamental para optimizarlos y evitar los subsidios cruzados entre los productos de alto rendimiento a favor de los de bajo rendimiento. Así por ejemplo, cuando se utiliza como criterio de asignación de costes a los distintos productos o servicios las horas de mano de obra directa, los servicios que son más intensivos en mano de obra reciben proporcionalmente más costes. Los servicios más comunes parecen menos rentables de los que realmente son a pesar de estar más estandarizados; por el contrario, los más específicos parecen más rentables a pesar de que demandan unos mayores esfuerzos (Ruiz y Tirado, 2004b).

Ya se ha comentado que el proceso productivo de la empresa bancaria se desglosa en los subprocesos de: captación de fondos, colocación de fondos y prestación de servicios. En las operaciones correspondientes a cada uno de los subprocesos participan diversos factores, mediante una sucesión de tareas.

Como las unidades de medida de los diferentes factores y productos no son homogéneas, y desde el punto de vista económico la transformación productiva debe evaluarse como la transformación del valor económico, es necesaria la transformación de las unidades técnicas de los factores que intervienen en el proceso en sus equivalentes monetarios.

Así, el proceso de formación del coste se inicia con la medición y valoración de los bienes y servicios que se incorporan a la transformación productiva -clases de coste- y concluye con la determinación del valor de los outputs, los cuales constituyen portadores de coste que cumplen con la función acumuladora de las distintas clases de coste (Ibarrondo, 2002b).

Las tres fases consustanciales al proceso de formación del coste son: clasificación, localización e imputación. Atendiendo a una clasificación de los costes por naturaleza, éstos podrán ser directos e indirectos. Son directos los consumos de bienes y servicios identificados de forma natural con el objeto de cálculo y de los que se conoce a priori su incidencia en el portador del coste. Son indirectos los que se tienen que imputar a los portadores de coste según un criterio de reparto previamente fijado (De la Cuesta, 1996).

Una clasificación de costes aceptada en el negocio bancario es la que diferencia entre factores materiales (impresos, material de oficina, documentos), 
de personal (valoración del trabajo humano), suministros (bienes no almacenables en los que se da la coincidencia en el tiempo del gasto-compra con su consumo, como energía y agua), servicios exteriores (prestaciones realizadas por unidades de producción ajenas a la analizada, vigilancia, seguridad), los del equipo productivo (amortizaciones de los bienes estructurales) y el de los recursos financieros que supone la valoración del consumo de los recursos financieros requeridos en la función de intermediación.

Ahora bien, no es fácil asignar un precio convencional que traduzca en unidades monetarias el consumo de factores durante el ejercicio económico, sino que en función de condicionantes externos a la empresa y de su coste de oportunidad es muy posible que se incluyan sesgos de cierta subjetividad.

El coste de los materiales y de personal antes mencionado puede desglosarse en costes directos e indirectos, sin embargo, el de los suministros y servicios exteriores serán costes indirectos. La asignación de costes indirectos, los cuales cada vez tienen un mayor peso en los costes totales de la empresa, dada la mayor automatización de los procesos, son muchas veces imputados siguiendo un criterio tradicional, como podría ser el basado en el factor productivo mano de obra o superficie ocupada, lo que en ocasiones proporciona una información errónea a los niveles jerárquicos que deben tomar las decisiones de planificación y control.

\subsection{El coste de los centros de coste}

La asignación de estos costes indirectos a los productos requiere de una agrupación y análisis previo, que se realiza en los centros de coste (también denominados secciones), antes de incorporarse al coste de producción. AECA (1999) define los centros o secciones de coste como espacios físicos donde se llevan a cabo determinadas actividades relacionadas con el objetivo económico de la empresa, al cual se asocia una cuenta o estado contable que permite acumular los costes de los factores utilizados por dichas actividades.

Los centros de costes pueden ser clasificados en principales, los que participan directamente en el proceso de producción, y auxiliares ${ }^{9}$, los que realizan sus prestaciones en función de otros centros, de modo que sus costes deben ser repercutidos sobre los bienes y servicios obtenidos en el

9 Otra clasificación en bibliografía es la establecida entre centros primarios y de apoyo, respectivamente. 
proceso de transformación de los principales ${ }^{10}$.

Según Ibarrondo (2002a) la descomposición de una cooperativa de crédito en centros de coste puede llevarse a efecto diferenciando, junto a los oportunos centros auxiliares, tres centros (secciones) de coste principales: Explotación, Comercial y Administración, dado que la participación y escasa relevancia del área funcional de aprovisionamiento en estas entidades no justifica su consideración al nivel de centro de coste. La sección (centro) de Explotación estará integrada por la red de sucursales y cajeros automáticos y por los departamentos operacionales, mientras que la sección Comercial y la sección de Administración se nutren de los departamentos no operacionales cuya actividad incide en las respectivas áreas funcionales. Los centros auxiliares se identifican con las secciones de apoyo encuadradas en los departamentos no operacionales.

La sección de Explotación (equivalente a la sección de transformación habitual en las empresas industriales) está compuesta por aquellos lugares de coste vinculados al proceso productivo. Es decir, las oficinas operativas que además de intervenir en el proceso de asignación de costes son unidades de análisis básicas, en tanto que se pueden determinar los ingresos, costes y márgenes a ellas imputables. También los cajeros automáticos pues participan en el subproceso de prestación de servicios puros. Y con respecto a los centros de costes operacionales los integran los departamentos de Cartera, Dirección financiera, Análisis de inversiones, Seguimiento de Riesgos-Morosidad, Relaciones Interbancarias, Tesorería, Extranjero y Valores. Estos centros intervienen en operaciones de producción de algunos de los subprocesos, y su coste resulta identificable en la estructura de costes del portador final.

En lo que se refiere a la sección Comercial cabe identificar como centros de coste los departamentos de Marketing, Comunicación e Imagen, y el destinado a la gestión del Fondo de Educación y Promoción Cooperativa (FEP) ${ }^{11}$.

10 En una empresa industrial estos centros principales son según R. RodRíGuez (1999): logística, operaciones, mercadotecnia y ventas, y servicio, y los auxiliares: abastecimiento, desarrollo de tecnología, recursos humanos e infraestructuras. Otro autor, J.A. BRIMSON (1995) distingue 5 centros principales: marketing y ventas, producción y control de calidad, investigación y desarrollo, finanzas y administración, y apoyo a la producción y logística.

11 Del resultado económico del ejercicio (de los beneficios resultantes una vez cubierto las pérdidas) se destinará el 10\% como mínimo a la dotación del FEP. (art. 8.3.b, Ley 13/1989, de 26 de mayo de cooperativas de crédito). El FEP se destina a actividades que cumplan alguna de las siguientes finalidades: a) la formación y educación de sus socios y trabajadores en 
Por su parte la sección de Administración de una cooperativa de crédito se integrará por la Asamblea General, el Consejo Rector, la Dirección General, Recursos Humanos, Auditoría, Contabilidad, Planificación-Organización y Control de Gestión. Estos centros no intervienen de forma directa en ningún subproceso productivo de modo que su asignación se efectuará de forma global al resultado del periodo.

Los centros auxiliares de costes, que tienen la característica de ceder sus prestaciones a otras unidades orgánicas de la entidad, son los de Asesoría Jurídica, Correspondencia, Almacén de material, Mantenimiento de equipos y Gestión de inmuebles. El gráfico 1 muestra el organigrama de centros de coste, principales y auxiliares, en una cooperativa de crédito.

\section{GRÁFICO 1. Centros de coste en una cooperativa de crédito}

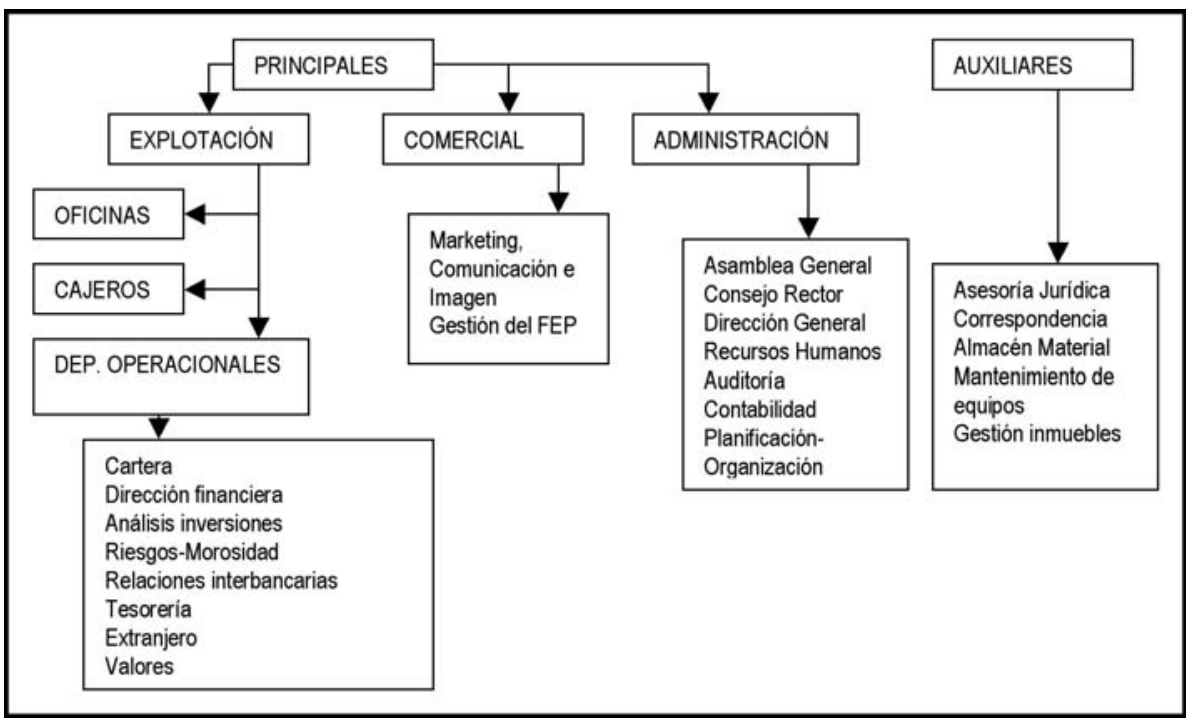

Fuente: Elaboración propia.

los principios y valores cooperativos, o en materias específicas de su actividad societaria o laboral y demás actividades cooperativas, b) la difusión del cooperativismo, así como la promoción de las relaciones intercooperativas, c) la promoción cultural profesional y asistencial del entorno local o de la comunidad en general, así como la mejora de la calidad de vida y del desarrollo comunitario y las acciones de protección medioambiental. (art. 56, Ley 27/1999, de 16 de julio, de cooperativas). 
Una vez identificados los centros de coste, habrá que imputar las diversas clases de costes según el consumo de factores y proceder al traspaso o reparto de los centros auxiliares a los principales, ya que son éstos últimos los que mantienen relaciones directas con los portadores. Algunos costes son fácilmente identificables y en otros se tendrá que proceder al reparto (servicios de seguridad, vigilancia, limpieza, valija, energía). Para medir la actividad de los centros auxiliares se podrá atender, dependiendo del centro al que haga referencia, a por ejemplo: $\mathrm{n}^{\mathrm{O}}$ horas de trabajo, $\mathrm{n}^{\mathrm{O}}$ consultas, $\mathrm{n}^{\mathrm{O}}$ informes, $\mathrm{n}^{\circ}$ envíos, $\mathrm{n}^{\mathrm{o}}$ ordenadores en mantenimiento, volumen de operaciones gestionado, $\mathrm{m}^{2}$ ocupados, etc.

En las cooperativas de crédito, como empresas de servicios, distinguiremos entre los costes vinculados a la producción, los que se derivan del normal desarrollo de los subprocesos productivos y se corresponden con la valoración del consumo de los factores aplicados a las fases por las que se sucede la contratación y prestación del servicio, y los vinculados al tiempo, que son los costes en los que incurre la empresa para mantener su estructura administrativa y comercial.

A continuación se describe el proceso de formación del coste en los tres subprocesos anteriormente descritos del output bancario, iniciándolo por el subproceso de prestación de servicios puros, al estar estrechamente vinculado a los subprocesos de captación y colocación de fondos.

\subsection{El subproceso de prestación de servicios puros}

Las tareas implicadas en la prestación de productos de servicio puro a los clientes de las cooperativas de crédito se podrán desglosar en dos grupos distintos, contratación del servicio y prestación del servicio, en aquellas que sea necesario una contratación específica y el servicio no venga dado como consecuencia de un depósito o de un producto de activo, debiendo asignar los costes respectivos a cada grupo, acumulándolos en un portador de coste. Para asignar estos costes habrá que delimitar las unidades técnicas de medida de cada output identificado en este subproceso. En el cuadro 3, se adjuntan estas unidades de medida según propuesta de Ibarrondo (2002a). 
CUADRO 3. Unidades de medida de los outputs del subproceso de prestación de servicios

\begin{tabular}{|c|c|c|}
\hline \multirow[t]{2}{*}{ Producto de servicio puro } & \multicolumn{2}{|c|}{ Medida del output } \\
\hline & $\begin{array}{l}\text { Contratación del } \\
\text { servicio }\end{array}$ & $\begin{array}{l}\text { Prestación del } \\
\text { servicio }\end{array}$ \\
\hline $\begin{array}{l}\text { Mediación de cobros y } \\
\text { pagos }\end{array}$ & $\begin{array}{l}\mathrm{N}^{\mathrm{o}} \text { productos } \\
\text { contratados }\end{array}$ & № transacciones \\
\hline Créditos de firma & \multicolumn{2}{|c|}{ № transacciones equivalentes } \\
\hline $\begin{array}{l}\text { Alquiler de cajas de } \\
\text { seguridad }\end{array}$ & № cajas alquiladas & № transacciones \\
\hline $\begin{array}{l}\text { Tramitación de ayudas, } \\
\text { subvenciones y licencias }\end{array}$ & \multicolumn{2}{|c|}{ № tramitaciones } \\
\hline Informes comerciales & \multicolumn{2}{|c|}{ № informes emitidos } \\
\hline Depósito de valores & \multicolumn{2}{|c|}{ № transacciones } \\
\hline $\begin{array}{l}\text { Mediación en la emisión de } \\
\text { valores }\end{array}$ & $\begin{array}{l}\text { № emisiones } \\
\text { contratadas }\end{array}$ & № transacciones \\
\hline $\begin{array}{l}\text { Comercialización } \\
\text { productos financieros }\end{array}$ & \multicolumn{2}{|c|}{ № transacciones } \\
\hline
\end{tabular}

Fuente: Ibarrondo, 2002a.

La formación del coste de los outputs del subproceso tendrá lugar con la imputación del coste de los centros que participan en la contratación y prestación del servicio (oficinas, cajero automático y otros centros operacionales). La unidad de prestación elegida para medir la actividad de la oficina es el tiempo de trabajo humano y para el cajero automático el de utilización de los equipos informáticos. Por lo tanto, es fundamental estandarizar los tiempos medios o normalizados de las distintas tareas en la prestación del servicio.

En cuanto a los otros centros operacionales las unidades de medida de los centros Extranjero, Cartera, Relaciones Interbancarias, Valores, Medios de Pago, son el $n^{o}$ de transacciones, el $n^{\circ}$ de efectos gestionados, en carte- 
ra, en gestión de cobro, descontados, devueltos, $\mathrm{n}^{\circ}$ de títulos en depósito $\mathrm{y}$ transacciones, $\mathrm{n}^{\mathrm{o}}$ de tarjetas, etc., debiendo asignarse a éstos un tiempo normalizado de trabajo humano, y al centro Explotación, un tiempo de utilización del equipo informático.

Coste producto servicio $=$ coste contratación + coste prestación

Coste producto servicio $=$ coste oficina + coste cajero + coste otros centros operacionales

Coste producto servicio $=\sum_{j=1}^{n}$ coste unidad prestación $n_{j} \times n^{o}$ unidades
prestación $_{j}$

Cantidad de output: $\mathrm{n}^{\circ}$ transacciones, $\mathrm{n}^{\circ}$ cajas alquiladas

Coste unitario producto $=$ coste total $/$ cantidad output

\subsection{El subproceso de captación de fondos}

El coste de los productos de pasivo, el proceso de captación de fondos, se obtendrá por el sumatorio del coste financiero devengado por los recursos ajenos obtenidos (mercado interbancario y ahorro de las unidades con superávit), más el coste proporcional de los centros principales que participan en su captación.

En éste habrá pues un coste directo, el imputado al producto con base en la retribución del recurso captado por la entidad, vía intereses, comisiones a otros intermediarios, retribuciones en especie, cargas asimiladas, etc. periodificado, que es con relativa facilidad identificable, y un coste indirecto resultante de la participación de diversos centros o lugares en su obtención, sobre el cual se podrá establecer una descomposición entre el coste propio de contratación y el de prestación.

La determinación del coste de los productos de pasivo en la entidad financiera, coste de la unidad monetaria captada, permite establecer un comparativo con el ingreso de cada unidad monetaria invertida en los respectivos productos de activo, a fin de conocer la contribución de sus depósitos al resultado global de la entidad.

Generalmente en la contratación de los depósitos, a la vista o a plazo, intervendrá únicamente la oficina donde se efectúa el depósito, pero en las posteriores prestaciones vinculadas al mismo, intervendrán también otros 
centros operacionales como el de Explotación, por el envío de información periódica o si se centraliza la liquidación de intereses, o el de Tesorería, por la mediación de cobros y pagos. En el caso de cesiones temporales de activo, podrán intervenir los departamentos de Tesorería, Valores, Relaciones Interbancarias y Explotación por el mismo motivo. En el gráfico 2 se muestra la interrelación entre los centros de coste y productos en el subproceso de captación de fondos.

\section{GRÁFICO 2. Subproceso de captación de fondos}

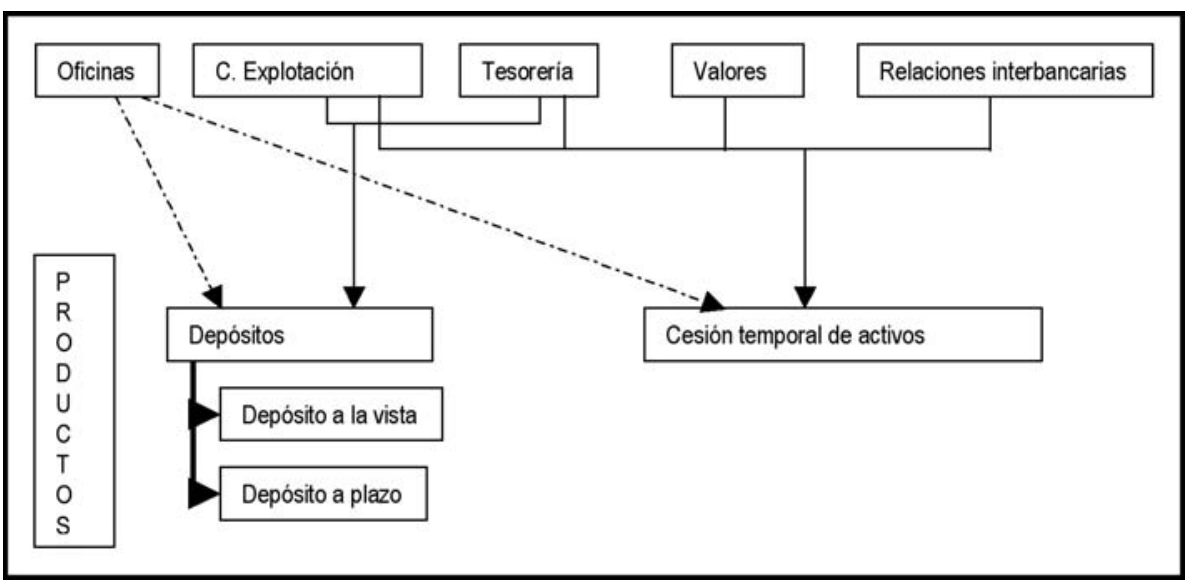

Fuente: Elaboración propia. Fase de contratación del producto en línea discontinua. Fase de prestación en línea continua.

La imputación del coste de oficinas podrá efectuarse según el tiempo de trabajo empleado (horas de mano de obra) en las tareas de contratación y prestación del producto, igual que en los centros operacionales (Valores, Tesorería, Relaciones Interbancarias) y el coste del centro de Explotación en función del tiempo empleado de los equipos informáticos en la contratación y prestación de cada producto de pasivo.

Coste producto pasivo = coste contratación + coste prestación

Coste contratación = coste financiero + coste indirecto (oficina, explotación, valores) 
Coste indirecto $=\sum_{\mathrm{j}=1}^{\mathrm{n}}$ coste unidad prestación $\mathrm{j}_{\mathrm{j}} \mathrm{xn}^{\mathrm{o}}$ unidades presta-
ción $_{\mathrm{j}}$

Coste unitario producto pasivo $=$ coste producto pasivo $/$ saldo medio periodo

\subsection{El subproceso de colocación de fondos}

En lo relativo al subproceso de colocación de fondos también se establece una subdivisión entre la contratación de los productos y las prestaciones vinculadas, como el envío de información al cliente, cargo de intereses, estudio de operaciones, etc. A su vez también es importante contemplar para cada producto, las operaciones en trámite de formalización, junto con las denegadas, pues se incurre en costes de estudio que no se trasladan a ingresos posteriores.

En la estructura del coste de los productos de activo, inversión crediticia y cartera de valores cabe distinguir entre el coste financiero de los fondos empleados para su colocación y el coste indirecto, consecuencia de la participación de los centros principales. Estos centros que intervienen son las oficinas, donde se efectúa la contratación del producto, el centro Valores que gestiona la inversión en valores, Dirección financiera por los estudios y autorización de operaciones, Análisis de inversiones, Seguimiento de Riesgos-Morosidad, por su estrecha vinculación con la concesión de créditos y posterior estudio de su evolución, Relaciones Interbancarias, por la gestión de cobros y pagos, Medios de Pago por las tarjetas de crédito, y Explotación por la centralización de algunas operaciones. La interrelación entre los centros de coste y los productos en el subproceso de colocación de fondos se muestra en el gráfico 3. 


\section{GRÁFÍCO 3. Subproceso de colocación de fondos}

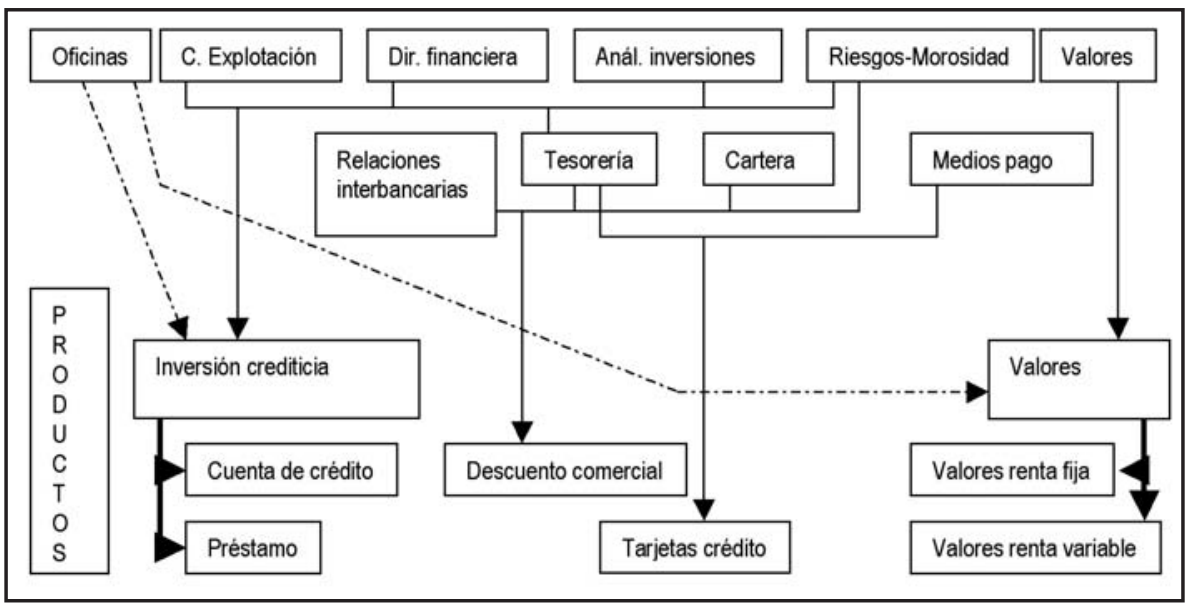

Fuente: Elaboración propia. Fase de contratación del producto en línea discontinua. Fase de prestación en línea continua.

Con respecto al estudio del coste financiero de cada producto de activo es fundamental calcular una tasa de transferencia interna (TTI) para los fondos, ya que captada una unidad monetaria es muy difícil identificarla con la unidad monetaria invertida en los productos de activo. El cálculo de esa tasa de transferencia puede efectuarse a través del denominado pool de fondos, el cual permite su cuantificación según se trabaje con base en un pool de fondos único o en un pool de fondos múltiple, y según se asigne sobre el cálculo de flujos brutos o netos.

A pesar de que es más riguroso el cálculo del pool múltiple de fondos brutos (De la Cuesta, 1996), las cooperativas de crédito suelen trabajar sobre la base del pool único de fondos netos, y esto es debido a dos motivos (Ibarrrondo, 2002a): en primer lugar por su simplicidad, pues la idea que subyace es que cualquier fuente de recursos puede financiar cualquier producto de activo, no siendo necesario identificar biunívocamente los recursos con los empleos, lo cual en algunos casos no está exento de cierta arbitrariedad, y en segundo lugar, porque cuando la entidad es de menor tamaño, y en las cooperativas de crédito esto ocurre, así como cuando los recursos financieros son relativamente estables, lo que también sucede pues se trabaja 
con una base importante de depósitos en su pasivo, no compensa el coste del cálculo de la tasa de transferencia según el sistema del pool múltiple de fondos con las ventajas de su utilización.

Con el pool único de fondos se considera que todos los recursos obtenidos son puestos en común y a partir de este fondo común la entidad financia sus operaciones. Y puesto que se trabaja sobre fondos netos los recursos transferidos entre las diversas oficinas se suponen exclusivamente ligados a los diferenciales entre recursos captados y recursos invertidos de cada una. En un estudio realizado por Ibarrondo (2002b) a las cajas rurales provinciales españolas o de ámbito superior, el 78\% de las cajas encuestadas (28 sobre una población objetivo de 34) aplicaban el método de flujos netos y el $85 \%$ tasa única (misma tasa para todos los fondos transferidos, tomando como referencia en su mayoría el precio de mercado).

En el cálculo de TTI se deben incluir los costes de naturaleza financiera de los productos de pasivo más los costes de explotación por los servicios prestados en la captación del ahorro, salvo que se puedan recuperar vía comisiones al conceder los créditos u otro producto derivado de la colocación de fondos.

Coste TTI $=$ (coste financiero captación + coste explotación (contratación

+ prestación servicio)) / recursos disponibles para inversión

El coste de los productos de activo será el que se derive de la suma del coste financiero más el coste de explotación. El coste financiero de los diferentes productos de activo, inversión crediticia: préstamos, cuentas de crédito y descuento comercial, así como cartera de valores se obtendrá por la aplicación de la tasa obtenida (TTI) por el capital pendiente de amortizar, por el saldo dispuesto según periodos, por el efectivo entregado debidamente periodificado o por la aplicación de la TTI a los fondos colocados en dichos títulos respectivamente.

En el proceso de formación de coste de los diferentes productos de activo hay que imputar la parte de costes indirectos de los centros de coste que intervienen en su contratación y prestación. Así pues, la oficina interviene en la contratación de la inversión crediticia y los centros operacionales Dirección financiera, Análisis de inversiones y Cartera en el caso del descuento de efectos. En la prestación y seguimiento posterior, la Oficina, Seguimiento de Riesgos-Morosidad, junto con el centro Explotación, por el envío de documentación. En el caso de descuento comercial, también intervienen Cartera y Relaciones Interbancarias. 
Las unidades de prestación para medir la actividad en las oficinas, Cartera, Relaciones Interbancarias y Explotación serán las mismas que en los subprocesos anteriormente descritos de prestación de servicio puro y captación de fondos. En el caso de los otros centros, Dirección financiera, Análisis de Inversiones, Seguimiento de Riesgos-Morosidad, puede efectuarse en función del tiempo de trabajo asignado a la operación, con lo que previamente se deberá proceder a la estandarización y normalización de tiempos por tareas.

Coste producto activo = coste contratación + coste prestación

Coste contratación = coste financiero + coste indirecto (oficina, explotación, valores)

Coste indirecto $=\sum_{j=1}^{n}$ coste unidad prestación $n_{j} x^{o}$ unidades prestación $_{j}$

Cantidad de output: saldo medio; $\mathrm{n}^{\circ}$ operaciones

Coste unitario producto activo $=$ coste producto activo $/$ cantidad output

\section{Conclusiones}

Los sistemas de contabilidad de gestión basados en las actividades cobran cada vez más importancia en un entorno como el financiero caracterizado por una fuerte competencia. Es importante que las entidades bancarias y en particular las cooperativas de crédito, dispongan de un sistema de asignación de costes que permita conocer la evolución del negocio y la contribución de los distintos productos a la generación del resultado de la empresa.

La contabilidad de gestión es una herramienta básica para la toma de decisiones en la empresa pues permite la planificación de actividades con base en los resultados sobre el coste de los productos, evitando los subsidios cruzados entre los diversos productos de la empresa. El proceso de transformación input-output en una cooperativa de crédito es complejo, no únicamente por la diversidad de sus outputs, la naturaleza multifásica, heterogeneidad, o el suministro de productos interdependientes, sino por el carácter intangible de algunos de ellos. La definición de actividades como herramienta de gestión, planificación y control para la toma de decisiones en la organización favorece la comprensión del funcionamiento de la misma, el porqué de las cosas y las relaciones causa-efecto. 
En las cooperativas de crédito las oficinas se perfilan como el elemento básico para el estudio de costes. Vinculado con el proceso de transformación económica que en el caso de las entidades financieras y dadas las peculiaridades del output bancario se establecen en los siguientes tres subprocesos: captación de fondos, colocación de fondos y prestación de servicios puros, se desarrolla el proceso de formación del coste que comprenderá el coste de contratación y el coste de prestación, y donde se verán implicados otros centros operacionales.

Un tema actual de enorme interés en prácticamente todos los ámbitos de la sociedad, es el de la Responsabilidad Social Corporativa (RSC), que responde a un modelo de gestión en el que trasciende a los beneficios puramente económicos, otros aspectos fundamentales como son los sociales y medioambientales. En este sentido las cooperativas de crédito tienen una magnífica oportunidad pues se da una coincidencia entre los principios y valores cooperativos que le son propios, con los requerimientos de la responsabilidad social. La consideración de las personas y el capital intelectual como activo fundamental en la empresa, la innovación, la oferta de servicios a los socios y clientes, la importancia de los aspectos intangibles y otras variables más allá del coste, son factores clave en la gestión de empresas y en los criterios de valoración que sobre éstas tienen los grupos de interés. Esto, por tanto, requiere del establecimiento de programas que definan las actividades que aporten elementos de valor para la RSC de las cooperativas de crédito.

Los modelos de gestión $\mathrm{ABC}-\mathrm{ABM}$ tienen un reto en la definición de actividades para establecer sobre ellas indicadores intangibles en línea con los nuevos referentes basados en criterios de RSC para la valoración de empresas. 


\section{Bibliografía}

AECA (1993), Costes indirectos de producción: localización, imputación y control. Serie Principios de Contabilidad de Gestión, documento $n^{\circ} 3$, Madrid, AECA.

- (1995), La contabilidad de gestión en las entidades bancarias. Serie Principios de Contabilidad de Gestión, documento n⿳⺈ 9, Madrid, AECA.

- (1998), El sistema de costes basado en las actividades. Serie Principios de Contabilidad de Gestión, documento $n^{0} 18$, Madrid, AECA.

- (1999), La contabilidad de gestión en las empresas agrarias. Serie Principios

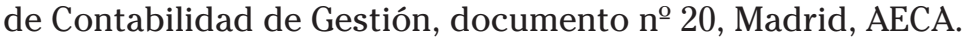

Амат, J. M. (1992), El control de gestión, una perspectiva de dirección, Barcelona, Ediciones Gestión 2000.

Banco de España (2006), Boletín Estadístico del Banco de España, http://www. bde.es

Barea, J., Monzón, J. L. (2002), La economía social en España en el año 2000, Valencia, Ed. Ciriec-España.

Barroso, C. (1995), Marketing bancario. Un enfoque estratégico, Madrid, ESIC.

Bengoechea, J., PizarRo, J. (1993), "Las nuevas fronteras del negocio bancario". Papeles de Economía Española, 54, 85-111.

Benston, G. J. (1972), "Economies of scale of financial institutions", Journal of money, credit and banking, may 312-341.

Blanco, F. (2002), Contabilidad de costes y analítica de gestión para las decisiones estratégicas, $9^{\text {a }}$ edición, Bilbao, Ediciones Deusto.

Brimson, J. A. (1995), Contabilidad por actividades, Barcelona, Marcombo Boixareu Editores.

Castelló, E., Lizcano, J. (1994), El sistema de gestión y de costes basado en las actividades, Madrid, Instituto de Estudios Económicos.

Chaves, R., SOLER, F. (2004): El gobierno de las cooperativas de crédito en España, Valencia, Ed. Ciriec-España.

CuesTA, M. DE LA (1996), El control de gestión en las entidades bancarias. Los modelos tradicionales y el ABM, Madrid, UNED. 
Fanjul, O., Maravall, F. (1985), La eficiencia del sistema bancario español, Madrid, Alianza.

FoRREST, E. (1996), Activity-based Management. A comprehensive implementation guide, Madrid, Ed. McGraw-Hill.

Fuentelsaz, L., Gómez, J. (2001), "La contabilidad de gestión en las cajas de ahorros". Cuadernos de Información Económica, 164, 104-112.

Fuentelsaz, L., Gómez, J., Lucea, V. (2003), "Las cooperativas de crédito en Aragón". Revista de Estudios Cooperativos, 79, 59-83.

García, F. J. (2001), "El sistema de costes ABC aplicado a una entidad financiera". Banca y finanzas, 62, 12-15.

García-Gutiérrez, C. (1995), "Las sociedades cooperativas de derecho y las de hecho con arreglo a los valores y a los principios del Congreso de la Alianza Cooperativa Internacional de Manchester en 1995: especial referencia a las sociedades de responsabilidad limitada reguladas en España". Revista de Estudios Cooperativos, 61, 53-88.

Grande, I. (1996), Marketing de servicios, Madrid, ESIC.

IBARRONDO, P. (2002a), Cálculo, análisis y control de costes en las cajas rurales, coedición Editorial Universidad de Granada y CIRIEC-España.

- (2002b), "La contabilidad de gestión en las cajas rurales. Una perspectiva empírica", CIRIEC-España, 41, 235-275.

- (2002c), "La producción de servicios bancarios. Problemática en torno a su delimitación”, Boletín Económico del ICE, 2735, 31-41.

KotLeR, P. (1992), Dirección de marketing, Londres, Prentice Hall.

LoRino, P. (1993), El control de gestión estratégico. La gestión por actividades, Barcelona, Marcombo Boixareu Editores.

Mackara, W. F. (1975), "What do banks produce?", Monthly Review Federal Reserve Bank of Atlanta, Vol. 60, 70-75.

Martinson, O. B. (1994), Cost Accounting in the Service Industry. A critical assessment, New Jersey, IMA, Montvale.

Mateos, A. (2002), Los costes y su sistema de control para la gestión de las empresas de comercialización citrícola. Estudio metodológico, Tesis Doctoral, Universidad Politécnica de Valencia. 
Morales, A. C. (2005), "Una tipología sociológica del cooperativismo: aplicación a diversos casos a nivel internacional", Revista de Fomento Social, 240, Vol. 60, 561-588.

Novo, J. A., VARela, R. (1996), Fundamentos económicos de la empresa bancaria. Universidad de La Coruña.

Palomo, R. J., VAlOR, C. (2001), Banca cooperativa. Entorno financiero y proyección social, Madrid, Unión Nacional de Cooperativas de Crédito, UNACC.

Pedelty, M. (1999), "Capital, democratisation and Governance”. International Co-operative Banking Association, 11, 5-18.

Perdiguer, M. (1991), "Productividad y calidad en los servicios financieros". Estrategia financiera, 65, 8-12.

PÉREZ, F., PASTOR, J. M. (1994), "La productividad del sistema bancario español”. Papeles de Economía Española, 58, 62-86.

PÉREZ, F., Quesada, J. (1991), Dinero y sistema bancario. Teoría y análisis del caso español, Madrid, Espasa-Calpe.

Prior, D., SAlas, V. (1994), "La eficiencia técnica de las cajas de ahorro y sus factores determinantes", Papeles de Economía Español, 58, 141-160.

QuesadA, J. (1994), Competividad y eficiencia de las entidades financieras: análisis comparativo. Perspectivas del sistema financiero, 48, 111-122.

Revell, J. (1988), "Las fusiones y el papel de los grandes bancos". Papeles de Economía Española, 36, 90-112.

RodríGuez, R. (1999), Costes por actividades en empresas de distribución minoristas, Barcelona, Marcombo Boixareu Editores.

Rodríguez, L., Parejo, J. A., Cuervo, A., Calvo, A. (2003), Manual del sistema financiero español, Barcelona, Ariel (16 edición, actualizada y revisada).

Ruiz, M., TIRADO, P. (2004a), “¿Como diseñar un sistema de costes basado en las actividades ABC? Un caso práctico", Técnica Contable, 667, 4-24.

- (2004b), "La gestión basada en las actividades en las entidades no lucrativas", Partida Doble, 158, 72-87.

Ś́nchez Asiain, J. A. (1992), Reflexiones sobre la banca, Madrid, Espasa Calpe.

Sealey, C. W., Lindley, J. T. (1977), "Inputs, outputs and theory of production and cost at depository financial institutions". The Journal of Finance, 4, $1251-1266$. 
TIRADO, P. (2003), Gestión de costes y mejora continua. Los sistemas de costes $y$ de gestión basados en las actividades ( $A B C-A B M)$, Colección ETEA, Ed. Desclée de Brouwer.

Unacc, (2005), Anuario de las Cajas Rurales, Populares y Profesionales, Madrid, Ed. Unión Nacional de Cooperativas de Crédito, UNACC.

VERA, S. (1997), El proceso de formación, asignación y captación del coste en el marco de la contabilidad interna, Monografía de Contabilidad de costes y de gestión, $\mathrm{n}^{\circ} 2$, Dep. Economía Financiera y Contabilidad, Universidad de Granada. 